\title{
Novel predictors of outcome after coronary angioplasty with rotational atherectomy. Not only low ejection fraction and clinical parameters matter
}

\author{
Piotr Kübler ${ }^{1,2}$, Wojciech Zimoch ${ }^{1,2}$, Michał Kosowski ${ }^{1,2}$, Brunon Tomasiewicz ${ }^{1,2}$, Oscar Rakotoarison ${ }^{2}$, \\ Artur Telichowski ${ }^{1,2}$, Krzysztof Reczuch ${ }^{1,2}$ \\ ${ }^{1}$ Military Hospital, Wroclaw, Poland \\ ${ }^{2}$ Department of Heart Diseases, Wroclaw Medical University, Wroclaw, Poland
}

Adv Interv Cardiol 2018; 14, 1 (51): 42-51

DOI: https://doi.org/10.5114/aic.2018.74354

\begin{abstract}
Introduction: Most established risk factors after rotational atherectomy (RA) of heavily fibro-calcified lesions are associated with patients' general risk and clinical related factors and are not specific for either coronary and culprit lesion anatomy or the RA procedure.

Aim: To assess novel predictors of poor outcome after percutaneous coronary intervention using RA in an all-comers population.

Material and methods: A total of 207 consecutive patients after RA were included in a single-center observational study. Primary endpoints were 1-year mortality and 1-year major adverse cardiac events (MACE). Secondary endpoints were angiographic and procedural success and in-hospital complications.

Results: Procedural complications occurred in 19 (8\%) patients. In-hospital mortality was 1\%, peri-procedural myocardial infarction (MI) was $9 \%$, and acute stroke occurred in one patient. The 1 -year MACE rate was $20 \%$ with all-cause mortality $10 \%, \mathrm{MI}$ $10 \%$ and stroke $1 \%$. Multivariable analysis revealed heart failure with left ventricle ejection fraction (LVEF) $\leq 35 \%(p=0.02)$ and uncrossable lesion, as compared to undilatable lesion $(p=0.01)$, as independent predictors of 1 -year mortality and residual SYNTAX score $\leq 8$ as an independent predictor of favorable outcome $(p=0.04)$. Heart failure with LVEF $\leq 35 \%(p<0.01)$ and uncrossable lesion $(p=0.04)$ were independent predictors of 1 -year MACE.

Conclusions: The presence of a novel factor, uncrossable lesion, as compared to undilatable lesion, is associated with poor outcome, and low residual SYNTAX score $\leq 8$ is associated with favorable outcome in 1-year follow-up after the RA procedure and can help in risk stratification of patients undergoing complex coronary intervention with RA.
\end{abstract}

Key words: rotablation, calcified lesion, undilatable lesion.

\section{Introduction}

Percutaneous coronary interventions $(\mathrm{PCl})$ are performed in more and more difficult settings nowadays, including heavily calcified and fibrotic lesions. In many patients rotational atherectomy (RA), along with orbital and excimer laser atherectomy, is the last resort for successful $\mathrm{PCl}$ of such highly calcified or fibrotic lesions $[1,2]$. According to European and American recommendations RA is indicated for calcified or massive fibrotic plaque modification for subsequent full balloon dilatation and optimal stent implantation [3, 4].

Since the introduction of RA to clinical practice 30 years ago, the procedure has been performed in a small number of patients and the number of trials and registries analyzing the outcome after RA has been modest, including mainly small and moderate-risk populations [5-9]. In recent years we have observed a constant increase in the number of RA procedures, which now constitute $1-3 \%$ of all $\mathrm{PCl}$ [3]. The results of new larger studies concerning this issue have also been published [10-15]. However, there is still a paucity of data regarding the outcome and proper risk stratification, taking into account the growing treatment needs of an overall aging population. Moreover, most of the established risk factors are associated with patient general risk and clinical related factors (including left ventricle ejection fraction (LVEF), diabetes mellitus, chronic kidney disease, and peripheral artery

\section{Corresponding author:}

Dr. Piotr Kübler, Department of Heart Diseases, Wroclaw Medical University, Military Hospital, 5 Weigla St, 50-981 Wroclaw, Poland, phone: +48 7176604 52, e-mail: pkubler75@gmail.com

Received: 4.12.2017, accepted: 10.12.2017. 
disease [13-15]) and are not specific for either coronary and culprit lesion anatomy or the RA procedure (like the use of drug-eluting stents (DES) [13]).

\section{Aim}

The aim of the study was in-hospital and 1-year outcome evaluation of patients after RA of fibro-calcified coronary lesions in an all-comers population including acute coronary syndromes (ACS), high-risk subjects and patients disqualified from coronary artery bypass grafting $(C A B G)$. Consequently, predictors of mortality and morbidity were determined, focusing on novel anatomy, lesion related and RA procedure related factors along with contemporary cardiovascular risk scores in order to help in better risk stratification in patients undergoing RA.

\section{Material and methods}

\section{Study population}

All consecutive patients with calcified coronary lesions who underwent $\mathrm{RA}$ and $\mathrm{PCl}$ in our institution from April 2008 to October 2015, regardless of indication and clinical status, were included in a single-center observational study. There were no exclusion criteria. Baseline demographic, clinical characteristics and detailed procedural data were collected, including indication for procedure, urgency, access site, all PCI equipment and lesion characteristics with basic quantitative coronary angiography (QCA) parameters. Preprocedural disqualification from CABG was undertaken by the local Heart Team. Information on all complications after each intervention, in-hospital and outcome major adverse cardiovascular events (MACE) was collected as well. All patients gave informed consent for the procedure. Follow-up data regarding all-cause mortality and recurrent hospitalizations and MACE were obtained from the Polish National Health Found database; therefore no patient was lost to follow-up. The study protocol was accepted by the local ethics committee and was in accordance with the Declaration of Helsinki.

\section{Study definitions}

An undilatable lesion indicated that the lesion that could not be adequately dilated by a balloon during inflation while an uncrossable lesion indicated that the lesion could be crossed by a wire, but could not be crossed with even the smallest balloons. The clinical risk was assessed according to logistic EuroSCORE $\|$ and the baseline SYNTAX score (SS) along with residual SS. SYNTAX score was calculated by two interventional cardiologists and in case of inconsistency a third calculation was done by the supervisor cardiologist. Residual SS $\leq 8$ was defined as low risk. The SYNTAX revascularization index (SRI) was calculated using the formula: SRI $=(1-$ [residual SS/ baseline SS]) $\times 100$ [16]. The modification of diet in renal diseases (MDRD) formula was used to calculate estimated glomerular filtration rate (eGFR).

\section{Definitions of endpoints}

Primary endpoints were 1-year mortality and 1-year MACE defined as the composite endpoint of all-cause mortality, follow-up myocardial infarction (MI) and stroke. Secondary endpoints were angiographic and procedural success and in-hospital complications. Follow-up MI and periprocedural MI were defined according to the universal definition of myocardial infarction [17]. Angiographic success was defined as residual stenosis of $<30 \%$ after stent implantation with thrombolysis in myocardial infarction (TIMI) flow grade III. Procedural success was defined as angiographic success without periprocedural complications.

\section{Procedure}

The RA procedure was performed using a standard Boston Scientific Rotablator system (Boston Scientific, Marlborough, MA, USA). The radial or femoral route was used according to operator discretion. Burr speeds were between 140,000 and $180,000 \mathrm{rpm}$ with a run duration to about 20-30 s. In all procedures an intracoronary continuous infusion of heparin, verapamil and isosorbide dinitrate via the burr sheath was used. Heparin was given to maintain an activated clotting time $>250 \mathrm{~s}$. All patients were pretreated with aspirin and clopidogrel, except 3 patients treated with ticagrelor and 1 treated with prasugrel. In-hospital treatment before and after RA was conducted according to current standards, including statins, $\beta$-blockers, angiotensin converter enzyme inhibitors and aldosterone antagonists and diuretics as necessary in patients with low LVEF and was left to the discretion of physicians in charge of the patients. Patients with atrial fibrillation were adequately anticoagulated and regulated according to current guidelines, including default periods of triple therapy, bridge therapy and antiarrhythmic drugs.

\section{Statistical analysis}

Continuous variables with normal distribution are presented as mean \pm standard deviation, continuous variables with skewed distribution as median with interquartile range and categorical variables as numbers and percentages. Univariate and multivariate Cox proportional hazard models were used to determine the predicting factors of all-cause death and composite endpoint (MACE). The multivariate model included all variables with $p<0.05$ in the univariate model. Survival and eventfree survival curves were created using the Kaplan-Meier method. Differences in survival and event-free survival rates were compared using the log-rank test. A $p$-value $<0.05$ was considered statistically significant. All statistical analyses were performed using the Statistica 10.0 (StatSoft, USA) software. 


\section{Results}

\section{Patient characteristics}

A total of 207 patients underwent $\mathrm{RA}$ and $\mathrm{PCl}$ during the study period. Mean age of the patients was $71.2 \pm 9.5$ years and 137 (66\%) were male. Complete demographics, numerous comorbidities and laboratory results are presented in Table I. Median logistic EuroSCORE II on admission was 2.4, median SS was 17 and low residual SS $\leq 8$ was present in 122 (59\%) patients. It is notewor-

Table I. Baseline clinical and laboratory characteristics

\begin{tabular}{|c|c|}
\hline Parameter & Value \\
\hline Patients & 207 \\
\hline Age [years] & $71.2 \pm 9.5$ \\
\hline Male & $137(66 \%)$ \\
\hline Body mass index $\left[\mathrm{kg} / \mathrm{m}^{2}\right]$ & $28.8 \pm 6.1$ \\
\hline Systolic blood pressure on admission [mm Hg] & $135 \pm 22$ \\
\hline Heart rate on admission [bpm] & $71 \pm 13$ \\
\hline Hypertension & $170(82 \%)$ \\
\hline Diabetes mellitus & $88(43 \%)$ \\
\hline Prior stroke/TIA & $26(13 \%)$ \\
\hline Hyperlipidemia & $96(46 \%)$ \\
\hline Thyroid disease & $28(14 \%)$ \\
\hline Cancer disease & $25(12 \%)$ \\
\hline Asthma/COPD & $14(7 \%)$ \\
\hline Current smoker & $15(7 \%)$ \\
\hline Atrial fibrillation & $42(20 \%)$ \\
\hline Peripheral artery disease & $64(31 \%)$ \\
\hline Severe valve disease & $19(9 \%)$ \\
\hline \multicolumn{2}{|l|}{ Left ventricle ejection fraction: } \\
\hline$\geq 50 \%$ & $137(66 \%)$ \\
\hline$<50 \%$ and $>35 \%$ & $35(17 \%)$ \\
\hline$\leq 35 \%$ & $35(17 \%)$ \\
\hline Impaired renal function with eGFR $<60 \mathrm{ml} / \mathrm{min}$ & $42(20 \%)$ \\
\hline Dialysis & $8(4 \%)$ \\
\hline Prior acute coronary syndrome & $130(63 \%)$ \\
\hline Prior PCl & $152(73 \%)$ \\
\hline Prior CABG & 31 (15\%) \\
\hline
\end{tabular}

thy that according to SS II the majority of patients (76\%) were potentially qualified to both treatment strategies (CABG or $\mathrm{PCl}$ ), but only $2 \%$ to $\mathrm{PCl}$ treatment alone.

\section{Procedure characteristics}

Fifty-one percent of patients underwent RA because of the presence of balloon undilatable lesions, $23 \%$ due to balloon uncrossable lesions and in $21 \%$ of patients RA was performed as a primary indication. A hundred

\begin{tabular}{lc} 
Parameter & Value \\
\hline Laboratory parameters: & \\
\hline White blood cell count $\left[\times 10^{3} / \mathrm{\mu l}\right]$ & $7.4(6.2-9.0)$ \\
\hline Red blood cell count $\left[\times 10^{6} / \mathrm{\mu l}\right]$ & $4.5(4.1-4.8)$ \\
\hline Hemoglobin $[\mathrm{g} / \mathrm{dll}]$ & $13.6 \pm 1.4$ \\
\hline Platelet count $\left[\times 10^{3} / \mathrm{\mu l}\right]$ & $205(176-249)$ \\
\hline Creatinine $[\mathrm{mg} / \mathrm{dl}]$ & $0.9(0.8-1.1)$ \\
\hline Glucose $[\mathrm{mg} / \mathrm{dl}]$ & $109(96-134)$ \\
\hline eGFR $\left[\mathrm{ml} / \mathrm{min} / 1.73 \mathrm{~m}^{2}\right]$ & $77 \pm 24$
\end{tabular}

\begin{tabular}{lc}
\hline Risk scores: & \\
\hline Logistic EuroSCORE II & $2.4(1.4-4.9)$ \\
\hline SYNTAX score & $17(11-24)$ \\
\hline Residual SYNTAX score & $8(0-14)$ \\
\hline Residual SYNTAX score $\leq 8$ & $122(59 \%)$ \\
\hline SYNTAX revascularization index & $58.8(42.1-100)$ \\
\hline SYNTAX score II - PCI & $5(2 \%)$ \\
\hline SYNTAX score II - CABG & $45(22 \%)$ \\
\hline SYNTAX score II - both & $157(76 \%)$
\end{tabular}

Medication at discharge:

\begin{tabular}{lc}
\hline Aspirin & $201(97 \%)$ \\
\hline P2Y12 inhibitor & $203(98 \%)$ \\
\hline$\beta$-Blocker & $195(94 \%)$ \\
\hline ACE inhibitor/ARB & $198(96 \%)$ \\
\hline Statin & $197(95 \%)$ \\
\hline Diuretic & $93(45 \%)$ \\
\hline Nitrates & $16(8 \%)$ \\
\hline Oral anticoagulation & $31(15 \%)$ \\
\hline Proton pump inhibitor & $100(48 \%)$
\end{tabular}

Data are presented as numbers and percentages for categorical variables, mean \pm standard deviation for continuous variables with normal distribution and median with interquartile range for continuous variables with skewed distribution. TIA - transient ischemic attack, COPD - chronic obstructive pulmonary disease, eGFR - estimated glomerular filtration rate, $P C I$ - percutaneous coronary intervention, $C A B G$ - coronary artery bypass grafting, ACE - angiotensin converter enzyme, $A R B$ - angiotensin receptor blocker. 
Table II. Procedure characteristics

\begin{tabular}{|c|c|}
\hline Characteristics & Value \\
\hline Acute coronary syndrome & $43(21 \%)$ \\
\hline Radial access & $125(60 \%)$ \\
\hline RA off label & $19(9 \%)$ \\
\hline Temporary pacing & 39 (19\%) \\
\hline IABP use & $4(2 \%)$ \\
\hline Inoperable patient: & $118(57 \%)$ \\
\hline Disqualified from CABG & $75(36 \%)$ \\
\hline No consent for CABG & $43(21 \%)$ \\
\hline \multicolumn{2}{|l|}{ Reason for RA: } \\
\hline Direct & $44(21 \%)$ \\
\hline Uncrossable & $48(23 \%)$ \\
\hline Undilatable & $105(51 \%)$ \\
\hline Unclear PCI failure & $10(5 \%)$ \\
\hline \multicolumn{2}{|l|}{ Target vessel: } \\
\hline RCA & $70(34 \%)$ \\
\hline LM & $11(5 \%)$ \\
\hline LAD & $93(44 \%)$ \\
\hline$C x$ & $37(17 \%)$ \\
\hline \multicolumn{2}{|l|}{ Lesion characteristics: } \\
\hline Lesion type B2/C & $186(90 \%)$ \\
\hline Aorto-ostial lesion & $27(13 \%)$ \\
\hline Bifurcation lesion & $81(39 \%)$ \\
\hline Chronic total occlusion & $23(11 \%)$ \\
\hline Severe calcifications & $186(90 \%)$ \\
\hline Diameter stenosis (\%) & $91 \pm 7$ \\
\hline Lesion length [mm] & $24(15-35)$ \\
\hline Minimum lumen diameter [mm] & $0.31 \pm 0.23$ \\
\hline Reference diameter [mm] & $3.1 \pm 0.5$ \\
\hline \multicolumn{2}{|l|}{ Procedural data: } \\
\hline Predilatation & $187(90 \%)$ \\
\hline Postdilatation & $108(52 \%)$ \\
\hline More than one burr & $39(19 \%)$ \\
\hline Burr to artery ratio & $0.45 \pm 0.07$ \\
\hline Maximum burr diameter & $1.5(1.25-1.5)$ \\
\hline Number of stents & $1.4 \pm 0.9$ \\
\hline DES implantations & $184(89 \%)$ \\
\hline Contrast volume [ml] & $266 \pm 97$ \\
\hline Fluoroscopy time [min] & $21(15-29)$ \\
\hline Procedure time [min] & $85(70-110)$ \\
\hline Radiation exposure [ $\mu \mathrm{Gy}]$ & $2623(1686-4171)$ \\
\hline Discharge after RA [days] & $2(1-4)$ \\
\hline Angiographic success & $203(98 \%)$ \\
\hline Procedural success & $192(93 \%)$ \\
\hline
\end{tabular}

Data are presented as numbers and percentages for categorical variables. mean + standard deviation for continuous variables with normal distribution and median with interquartile range for continuous variables with skewed distribution. RA - rotational atherectomy, IABP - intra-aortic balloon pump, $C A B G$ - coronary artery bypass grafting, $P C l$ - percutaneous coronary intervention, $R C A$ - right coronary artery, $L M$ - left main, $L A D$ - left anterior descending, $C x$ - circumflex artery, DES - drug-eluting stent. eighty-eight (57\%) patients were disqualified or did not consent to CABG. Angiographic success was $98 \%$ with procedural success $93 \%$. Complete procedure data are presented in Table II.

\section{In-hospital and 1-year outcome}

Procedural complications occurred in 19 (8\%) patients with no need for urgent CABG treatment. In-hospital mortality was $1 \%$, peri-procedural MI $9 \%$, and acute stroke in 1 patient before discharge occurred. The 1-year MACE rate was $20 \%$ with all-cause mortality $10 \%$, MI rate $10 \%$ and stroke $1 \%$ (Table III).

\section{Predictors of adverse events in 1-year follow-up}

Univariate analysis identified the following risk factors for 1-year all-cause death: heart failure with LVEF $\leq 35 \%(p<0.001)$, presence of an uncrossable lesion $(p<0.01)$, eGFR $<60 \mathrm{ml} / \mathrm{min}(p=0.04)$, and EuroSCORE II $(p<0.01)$. Residual SS $\leq 8$ was identified as favorable factor $(p<0.01)$. Multivariable analysis revealed heart

Table III. In-hospital and 1-year follow-up adverse events

\begin{tabular}{lc} 
Variable & Value \\
\hline Periprocedural complications: & $19(8 \%)$ \\
\hline Slow/no-flow & $3(1 \%)$ \\
\hline Side branch occlusion & $5(2 \%)$ \\
\hline Dissection & $8(4 \%)$ \\
\hline Perforation & $3(1 \%)$ \\
\hline Emergency CABG & $0(0 \%)$ \\
\hline Permanent pacing & $0(0 \%)$ \\
\hline In-hospital outcomes: & $2(1 \%)$ \\
\hline Death & $18(9 \%)$ \\
\hline Peri-procedural MI & $1(1 \%)$ \\
\hline Stroke/TIA & $1(1 \%)$ \\
\hline Target vessel revascularization & $6(3 \%)$ \\
\hline Contrast induced nephropathy & $14(7 \%)$ \\
\hline Access site bleedings & $3(1 \%)$ \\
\hline Access site interventions & $20(10 \%)$ \\
\hline Clinical outcomes at 1-year follow-up: & $42(20 \%)$ \\
\hline Death & $20 \%)$ \\
\hline Stroke & \\
\hline MACE & \\
\hline
\end{tabular}

Data are presented as numbers and percentages. CABG - coronary artery bypass grafting, MI - myocardial infarction, TIA - transient ischemic attack, MACE - major adverse cardiac events. 
Table IV. Predictors of all-cause death in Cox regression models

\begin{tabular}{|c|c|c|c|c|c|c|}
\hline \multirow[t]{2}{*}{ Parameter } & \multicolumn{3}{|c|}{ Univariate model } & \multicolumn{3}{|c|}{ Multivariate model } \\
\hline & HR & $95 \% \mathrm{Cl}$ & $P$-value & HR & $95 \% \mathrm{Cl}$ & $P$-value \\
\hline LVEF $\leq 35 \%$ & 5.64 & $2.34-13.55$ & $<0.001$ & 3.18 & $1.21-8.40$ & 0.02 \\
\hline Uncrossable lesion & 3.64 & $1.51-8.74$ & $<0.01$ & 3.43 & $1.34-8.80$ & 0.01 \\
\hline Residual SYNTAX score $\leq 8$ & 0.14 & $0.04-0.47$ & $<0.01$ & 0.25 & $0.07-0.92$ & 0.04 \\
\hline $\mathrm{eGFR}<60 \mathrm{ml} / \mathrm{min}$ & 2.58 & $1.05-6.31$ & 0.04 & 1.36 & $0.47-3.95$ & 0.57 \\
\hline EuroSCORE II & 1.09 & $1.03-1.15$ & $<0.01$ & 1.04 & $0.96-1.13$ & 0.30 \\
\hline Age & 1.04 & $0.99-1.10$ & 0.10 & & & \\
\hline Male & 1.18 & $0.45-3.06$ & 0.74 & & & \\
\hline Prior ACS & 0.70 & $0.29-1.70$ & 0.44 & & & \\
\hline Prior CABG & 0.99 & $0.29-3.38$ & 0.99 & & & \\
\hline Inoperable patient & 2.45 & $0.89-6.75$ & 0.08 & & & \\
\hline Hypertension & 0.64 & $0.23-1.75$ & 0.38 & & & \\
\hline Diabetes mellitus & 1.71 & $0.71-4.14$ & 0.23 & & & \\
\hline Prior stroke/TIA & 1.24 & $0.36-4.24$ & 0.73 & & & \\
\hline Hyperlipidemia & 0.95 & $0.40-2.29$ & 0.90 & & & \\
\hline Cancer disease & 0.38 & $0.05-2.81$ & 0.34 & & & \\
\hline Hemoglobin level & 0.27 & $0.60-1.14$ & 0.25 & & & \\
\hline SYNTAX score > 32 & 1.32 & $0.39-4.51$ & 0.66 & & & \\
\hline$S R I>50 \%$ & 0.47 & $0.19-1.13$ & 0.09 & & & \\
\hline Non-ACS & 0.56 & $0.22-1.46$ & 0.24 & & & \\
\hline RA off label & 2.64 & $0.88-7.89$ & 0.08 & & & \\
\hline Severe calcifications & 1.00 & $0.23-4.30$ & 1.00 & & & \\
\hline Radial access & 0.80 & $0.33-1.92$ & 0.61 & & & \\
\hline Lesion length & 1.00 & $0.98-1.03$ & 0.70 & & & \\
\hline Lesion B2/C & 0.99 & $0.99-1.02$ & 0.69 & & & \\
\hline Bifurcation & 0.84 & $0.33-2.10$ & 0.70 & & & \\
\hline Predilatation & 0.77 & $0.18-3.30$ & 0.72 & & & \\
\hline Postdilatation & 0.89 & $0.37-2.13$ & 0.79 & & & \\
\hline Number of burrs & 1.49 & $0.65-3.40$ & 0.35 & & & \\
\hline Maximal burr diameter & 0.58 & $0.04-7.41$ & 0.67 & & & \\
\hline DES implantation & 1.00 & $0.13-7.49$ & 1.00 & & & \\
\hline Burr to artery ratio & 2.70 & $0.47-15.20$ & 0.08 & & & \\
\hline Contrast volume & 0.99 & $0.99-1.01$ & 0.35 & & & \\
\hline Complications & 0.51 & $0.07-3.83$ & 0.51 & & & \\
\hline
\end{tabular}

$H R$ - hazard ratio, Cl - confidence interval, LVEF - left ventricle ejection fraction, eGFR - estimated glomerular filtration rate, ACS - acute coronary syndrome, CABG - coronary artery bypass grafting, TIA - transient ischemic attack, SRI - SYNTAX revascularization index, RA - rotational atherectomy, DES - drug-eluting stent. 
Table V. Predictors of major adverse cardiac events in Cox regression models

\begin{tabular}{|c|c|c|c|c|c|c|}
\hline \multirow[t]{2}{*}{ Parameter } & \multicolumn{3}{|c|}{ Univariate model } & \multicolumn{3}{|c|}{ Multivariate model } \\
\hline & $\mathrm{HR}$ & $95 \% \mathrm{Cl}$ & $P$-value & $\mathrm{HR}$ & $95 \% \mathrm{Cl}$ & $P$-value \\
\hline LVEF $\leq 35 \%$ & 3.25 & $1.76-6.02$ & $<0.001$ & 2.67 & $1.38-5.13$ & $<0.01$ \\
\hline Uncrossable lesion & 2.01 & $1.11-3.81$ & 0.02 & 1.89 & $1.01-3.55$ & 0.04 \\
\hline Residual SYNTAX score $\leq 8$ & 0.40 & $0.22-0.75$ & $<0.01$ & 0.64 & $0.32-1.27$ & 0.20 \\
\hline Age & 1.04 & $1.01-1.08$ & 0.01 & 1.03 & $0.99-1.07$ & 0.07 \\
\hline Diabetes mellitus & 1.93 & $1.06-3.49$ & 0.03 & 1.82 & $0.99-3.33$ & 0.06 \\
\hline EuroSCORE II & 1.07 & $1.03-1.12$ & 0.01 & 1.02 & $0.96-1.09$ & 0.45 \\
\hline $\mathrm{eGFR}<60 \mathrm{ml} / \mathrm{min}$ & 1.25 & $0.63-2.47$ & 0.52 & & & \\
\hline Male & 0.89 & $0.48-1.65$ & 0.71 & & & \\
\hline Prior ACS & 0.93 & $0.51-1.70$ & 0.81 & & & \\
\hline Prior CABG & 1.62 & $0.78-3.37$ & 0.19 & & & \\
\hline Inoperable patient & 1.46 & $0.79-2.69$ & 0.23 & & & \\
\hline Hypertension & 1.78 & $0.70-4.52$ & 0.22 & & & \\
\hline Prior stroke/TIA & 1.12 & $0.47-2.65$ & 0.79 & & & \\
\hline Hyperlipidemia & 0.97 & $0.54-1.76$ & 0.93 & & & \\
\hline Cancer disease & 1.79 & $0.83-3.85$ & 0.14 & & & \\
\hline Hemoglobin level & 0.89 & $0.71-1.11$ & 0.30 & & & \\
\hline SYNTAX score $>32$ & 1.03 & $0.60-1.50$ & 0.12 & & & \\
\hline $\mathrm{SRI}>50 \%$ & 0.99 & $0.88-1.11$ & 0.08 & & & \\
\hline Non-ACS & 0.55 & $0.29-1.08$ & 0.07 & & & \\
\hline RA off label & 2.18 & $0.97-4.89$ & 0.06 & & & \\
\hline Severe calcifications & 2.47 & $0.59-10.10$ & 0.22 & & & \\
\hline Radial access & 0.94 & $0.52-1.72$ & 0.85 & & & \\
\hline Lesion length & 1.01 & $0.98-1.02$ & 0.47 & & & \\
\hline Lesion B2/C & 0.68 & $0.29-1.62$ & 0.39 & & & \\
\hline Bifurcation & 0.97 & $0.54-1.81$ & 0.96 & & & \\
\hline Predilatation & 0.87 & $0.31-2.42$ & 0.78 & & & \\
\hline Postdilatation & 0.78 & $0.44-1.42$ & 0.43 & & & \\
\hline Number of burrs & 1.08 & $0.58-2.01$ & 0.80 & & & \\
\hline Max burr diameter & 0.46 & $0.08-2.60$ & 0.38 & & & \\
\hline DES implantation & 2.44 & $0.34-17.76$ & 0.38 & & & \\
\hline Burr to artery ratio & 6.96 & $0.09-56.18$ & 0.39 & & & \\
\hline Contrast volume & 1.00 & $0.99-1.01$ & 0.87 & & & \\
\hline Complications & 0.70 & $0.22-2.26$ & 0.55 & & & \\
\hline
\end{tabular}

$H R$ - hazard ratio, Cl - confidence interval, LVEF - left ventricle ejection fraction, eGFR - estimated glomerular filtration rate, ACS - acute coronary syndrome, CABG - coronary artery bypass grafting, TIA - transient ischemic attack, SRI - SYNTAX revascularization index, RA-rotational atherectomy, DES - drug-eluting stent. 
failure with LVEF $\leq 35 \%(\mathrm{HR}=3.18,95 \% \mathrm{Cl}: 1.21-8.40$, $p=0.02)$ and uncrossable lesion $(\mathrm{HR}=3.43,95 \% \mathrm{Cl}$ : $1.34-8.80, p=0.01)$ as independent predictors of 1-year mortality and residual $\mathrm{SS} \leq 8(\mathrm{HR}=0.25,95 \% \mathrm{Cl}: 0.07-$ $0.92, p=0.04$ ) as an independent predictor of a favorable outcome (Table IV).

Univariate analysis identified the following risk factors for follow-up MACE: heart failure with LVEF $\leq 35 \%$ $(p<0.001)$, presence of an uncrossable lesion $(p=0.02)$, age $(p=0.01)$, diabetes mellitus $(p=0.03)$, and EuroSCORE $\|(p=0.01)$. Residual SS $\leq 8$ was identified as a favorable factor $(p<0.01)$. Multivariable analysis again revealed heart failure with LVEF $\leq 35 \%(H R=2.67$, $95 \% \mathrm{Cl}: 1.38-5.13, p<0.01)$ and uncrossable lesion $(\mathrm{HR}=1.89,95 \% \mathrm{Cl}: 1.01-3.55, p=0.04)$ as independent predictors of 1-year MACE (Table V).

Kaplan-Meier curves were plotted to assess survival data for every independent factor: heart failure with LVEF $\leq 35 \%$, presence of an uncrossable lesion and residual $\mathrm{SS} \leq 8$ (Figure 1). Additional curves were plotted to assess

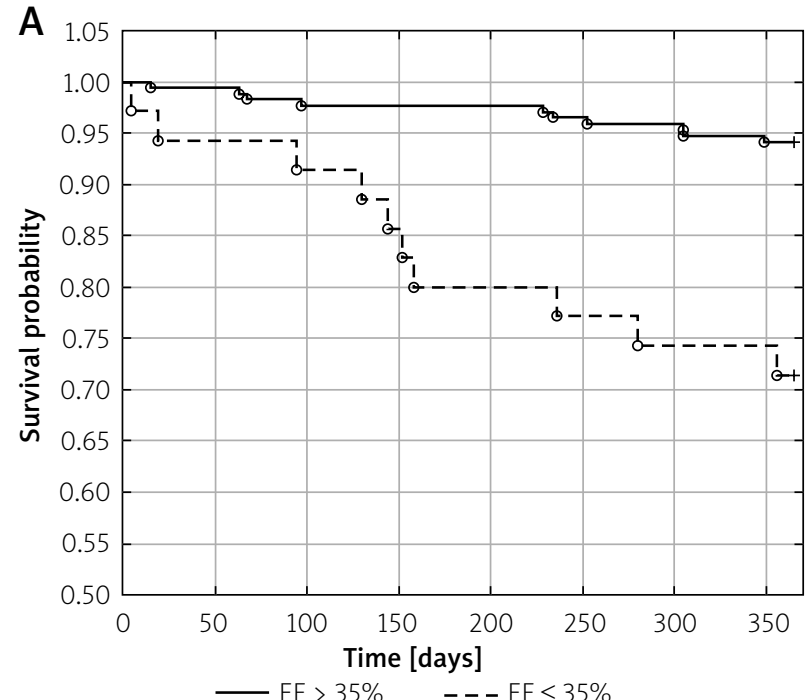

C

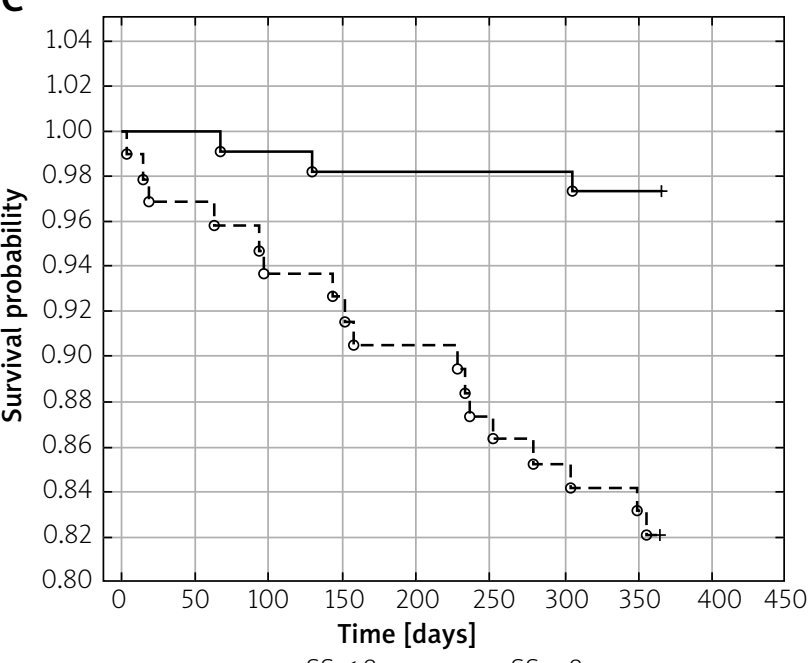

data for every independent risk factor of MACE: heart failure with LVEF $\leq 35 \%$ and presence of an uncrossable lesion (Figure 2). Values of the log-rank test comparison demonstrated a significantly decreased survival rate with either of the independent risk factors of mortality and a significantly decreased MACE-free survival rate for both independent risk factors of MACE, as compared with the rest of the study population.

\section{Discussion}

In our study we analyzed a high-risk all-comers population of patients with heavily calcified or fibrotic coronary lesions, who underwent RA procedure. The main findings of our study are: 1) we indicated two novel, anatomy and lesion related factors in the population undergoing RA - the presence of an uncrossable lesion, as compared to an undilatable lesion, was unfavorable, and low residual SS $\leq 8$ was favorable; 2 ) RA is a feasible and effective procedure even in patients without other revascularization options.

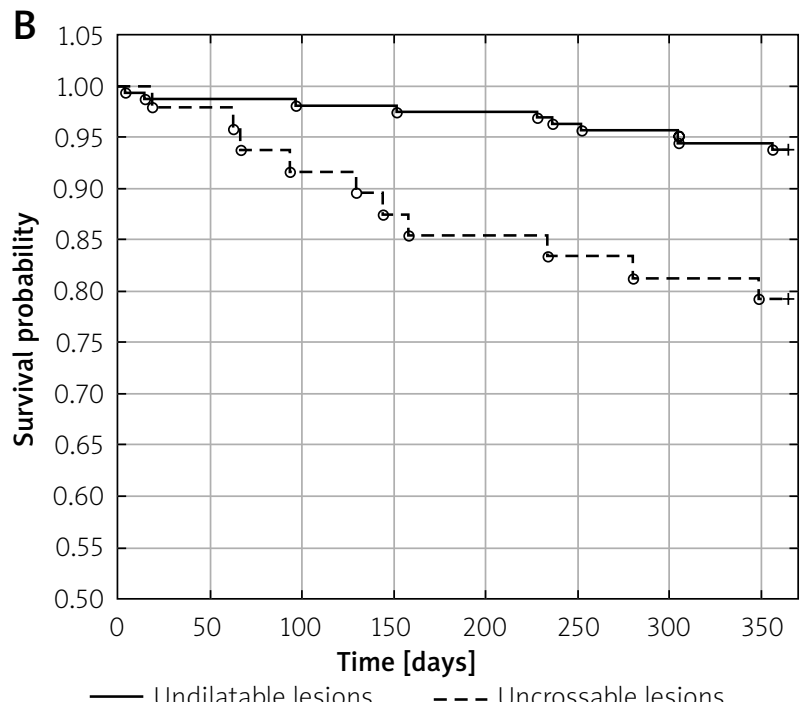

Figure 1. Kaplan-Meier survival curves according to left ventricle ejection fraction (EF), log rank $p<0.001(\mathrm{~A})$, according to type of lesion, log rank $p=0.003$ (B) and according to residual SYNTAX score (rSS), log rank $p<0.001$ (C) 
The studied population was all-comers without exclusions, with high cardiovascular risk associated with advanced age (mean: 71.2 years) and numerous comorbidities. Baseline risk scores were relatively high: median logistic EuroSCORE II was 2.4 and SS was 17. In contrast to earlier publications investigating moderate-risk patients, recent studies analyzed high-risk patients as well, with an estimated EuroSCORE II of 2.1 and a SYNTAX score of 19.5 [13-15]. The frequency of ACS in these recent studies reached $20-40 \%$, similar to our population (21\%), alongside similar frequency of type B2/C lesion according to the ACC/AHA classification reaching $90 \%$. It should be noted that a radial approach was used in $60 \%$ of our patients, compared to $30-50 \%$ in aforementioned studies. The median burr-to-artery ratio was relatively low $(0.45 \pm 0.07)$, which was our initial strategy and proved to be effective for plaque modification, in line with the European expert consensus on RA [3]. It is also noteworthy that $36 \%$ of our patients were disqualified from CABG and $21 \%$ of them gave no consent for surgery. It means we analyzed a real-world high-risk group of patients, with RA use as the last revascularization option. Nonetheless the angiographic success was $98 \%$ with procedure success $93 \%$, the periprocedural complication and MI rate was less than $10 \%$ and in-hospital mortality $1 \%$, which is comparable to other results [10-12]. Vascular access complications were relatively high, reaching $7 \%$, but almost all occurred with the femoral approach during the first years of performing RA. In 1-year follow-up the mortality rate was $10 \%$ and the MACE rate $20 \%$, which is comparable with other outcomes $[5,14,15]$. In a recent analysis the 1-year mortality and MACE rates were even lower, $5 \%$ and $16 \%$, respectively [13].

The presence of heart failure with decreased LVEF is a well-documented independent predictor of mortality,

A

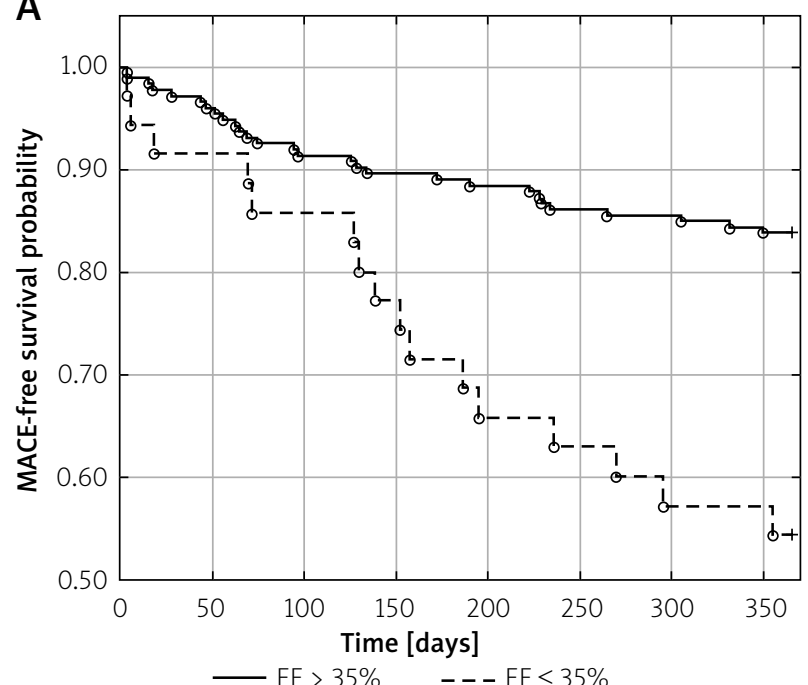

following not only a conventional $\mathrm{PCI}$ procedure but also $\mathrm{PCl}$ with accompanying $\mathrm{RA}[5,14,18]$. In our multivariable analysis heart failure with LVEF $\leq 35 \%$ was also an independent predictor of mortality $(p=0.02)$, and moreover an independent predictor of MACE $(p<0.01)$. Patients with decreased LVEF undergoing complex $\mathrm{PCl}$ along with RA could be candidates for more specialized treatment, such as the use of mechanical circulatory support. A couple of other, fairly general, clinical related predictors of poor outcome after RA were observed in the literature in particular studies, such as chronic kidney disease and dialysis [13, 14], diabetes mellitus [13-15], and peripheral vascular disease [15]. None of them proved to be an independent risk factor in our multivariable analysis.

However, we decided to analyze in our database more anatomical, lesion and procedure-related predictors, specific for RA. The second independent predictor of both mortality $(p=0.01)$ and MACE $(p=0.04)$ in the multivariable analysis was the presence of an uncrossable lesion, as compared to an undilatable lesion. The occurrence of a lesion that cannot be crossed by a balloon catheter and a lesion that cannot be adequately dilated by the balloon are two main indications to perform RA and occurred in $23 \%$ and $51 \%$ of our patients, respectively. The RA can also be performed as a primary indication, mainly on the basis of visible extensive calcifications in coronary angiography, which were present in $21 \%$ of our patients. Underlying pathophysiological reasons why uncrossable lesions, in comparison with undilatable lesions, are independently associated with poor outcome, are yet to be fully determined. One could hypothesize that the presence of such tough and simultaneously tight stenoses reflects a very advanced general atherosclerotic process in the human cardiovascular system. Furthermore, successful treatment of that lesion may require a more complex

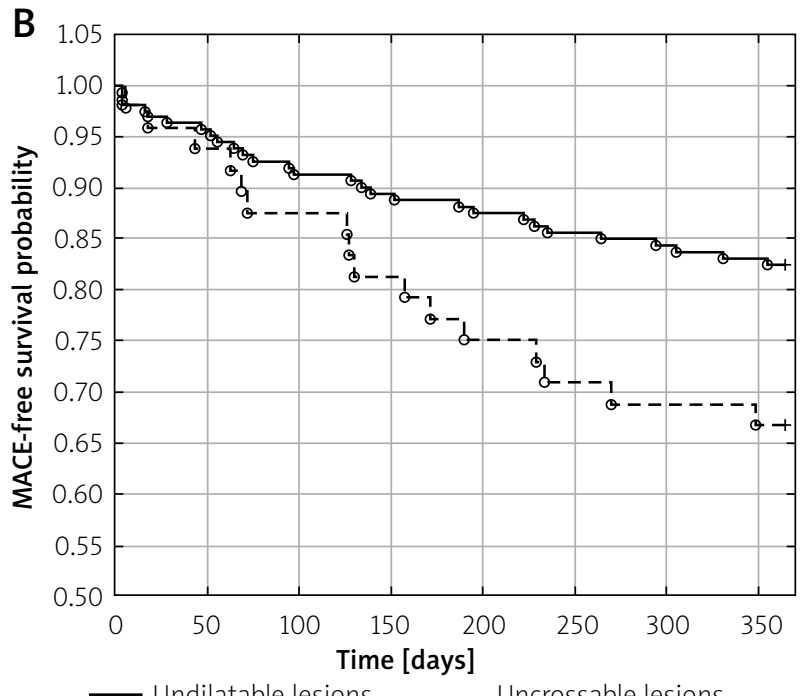

_ Undilatable lesions _- - Uncrossable lesions

Figure 2. Kaplan-Meier major adverse cardiac event (MACE)-free survival curves according to left ventricle ejection fraction (EF), log rank $p<0.001$ (A) and according to type of lesion, log rank $p=0.02$ (B) 
RA procedure, with Rota-wire passage that commonly is more complicated, with the use of more than one burr, more contrast media, longer and several burr passages and, consequently, may be associated with more complications and poorer outcome. We can find some similarities of such lesions with typical chronic total occlusions (CTO), although in the majority of RA cases antegrade flow is initially maintained. In data concerning CTO treatment balloon uncrossable lesions more often had calcifications and substantial tortuosity, required a longer procedure time and procedural success was significantly lower along with more periprocedural complications [19, 20]. Further larger studies evaluating causes of higher morbidity and mortality in this subpopulation are needed. Other procedure-related risk factors of poorer outcome after RA from the literature are the use of DES versus bare metal stent (BMS), total stent length [13], and triple-vessel disease (versus single-vessel disease) [12]. However, the latter predictors are not specific for the RA procedure.

Moreover, we analyzed contemporary widely used cardiovascular risk scores such as EuroSCORE II, which is based on clinical variables, and SS, based on coronary lesion anatomy. Neither final score was associated with outcome in our analysis. In contrast, in one multicentre RA registry $\mathrm{SS} \geq 23$ and in one single-center registry high EuroSCORE II had a significant association with major adverse cardiac events in long-term observation [15]. Additionally, the importance of incomplete revascularization after conventional $\mathrm{PCl}$ treatment and its influence on prognosis was raised in recent trials, indicating that residual SS > 8 was associated with poor prognosis [21-23]. We found that low residual $\mathrm{SS} \leq 8$ was an independent predictor of better survival $(p<0.01)$, which means that this level of incomplete revascularization may be useful in risk stratification also in a high-risk population undergoing RA. The SYNTAX revascularization index, also proposed for assessing reasonable incomplete revascularization, has not been shown to be a significant factor for the occurrence of death or adverse events in our population [16].

Therefore, we conclude that a comprehensive approach, including not only general and clinical related factors but also lesion related, RA related and coronary anatomy related factors can constitute a better risk stratification in this specific high-risk population undergoing RA. The presence of an uncrossable lesion and the lack of low residual SS, which can be indicated before the RA procedure, can help in identifying a higher risk subgroup, with the potential necessity of more advanced treatment. Larger studies are required to confirm the role of the abovementioned risk factors and to create a more standardized risk stratification protocol of patients qualifying for RA.

The study was an observational single-arm registry from a single high-volume center. The sample size and the number of events, particularly deaths, was relatively small. Regression analyses are, however, explorative. The use of stents was not quite uniform, with BMS use in $11 \%$ of patients. All-cause mortality was only reported without differentiating the group of cardiac death patients.

\section{Conclusions}

The presence of an uncrossable lesion, as compared to an undilatable lesion, is associated with poor outcome, and low residual SS $\leq 8$ is associated with a favorable outcome in 1-year follow-up after the RA procedure. The abovementioned predictors along with clinical risk factors including decreased LVEF can help in risk stratification of patients undergoing complex PCl with RA. Percutaneous coronary intervention with accompanying RA is a feasible and effective procedure even in high-risk patients without other revascularization options.

\section{Acknowledgments}

The publication has been financially supported by the statutory grant from the Polish Ministry of Science and Higher Education No. ST.E.190.16.066.

\section{Conflict of interest}

The authors declare no conflict of interest.

\section{References}

1. Fujii K, Carlier SG, Mintz GS, et al. Stent underexpansion and residual reference segment stenosis are related to stent thrombosis after sirolimus-eluting stent implantation. J Am Coll Cardiol 2005; 45: 995-8.

2. Im E, Kim BK, Ko YG, et al. Incidences, predictors, and clinical outcomes of acute and late stent malapposition detected by optical coherence tomography after drug-eluting stent implantation. Circ Cardiovasc Interv 2014; 7: 88-96.

3. Barbato E, Carrié D, Dardas P, et al. European expert consensus on rotational atherectomy. Eurolntervention 2015; 11: 30-6.

4. Tomey MI, Kini AS, Sharma SK. Current status of rotational atherectomy. JACC Cardiovasc Interv 2014; 7: 345-53.

5. Abdel-Wahab M, Baev R, Dieker P, et al. Long-term clinical outcome of rotational atherectomy followed by drug-eluting stent implantation in complex calcified coronary lesions. Catheter Cardiovasc Interv 2013; 81: 285-91.

6. Benezet J, Diaz de la Llera L, Cubero J, et al. Drug-eluting stents following rotational atherectomy for heavily calcified coronary lesions: long-term clinical outcomes. J Invasive Cardiol 2011; 23: 28-32.

7. Abdel-Wahab M, Richardt G, Joachim Büttner $\mathrm{H}$, et al. Highspeed rotational atherectomy before paclitaxel-eluting stent implantation in complex calcified coronary lesions: The randomized ROTAXUS (Rotational Atherectomy Prior to Taxus Stent Treatment for Complex Native Coronary Artery Disease) trial. JACC Cardiovasc Interv 2013; 6: 10-9.

8. Mezilis N, Dardas P, Ninios V, et al. Rotablation in the drug eluting era: immediate and long-term results from a single center experience. J Interv Cardiol 2010; 23: 249-53. 
9. Rathore $\mathrm{S}$, Matsuo H, Terashima M, et al. Rotational atherectomy for fibrocalcific coronary artery disease in drug eluting stent era: procedural outcomes and angiographic follow-up results. Catheter Cardiovasc Interv 2010; 75: 919-27.

10. Arora S, Panaich SS, Patel N, et al. Coronary atherectomy in the United States (from a Nationwide Inpatient Sample). Am J Cardiol 2016; 117: 555-62.

11. Cockburn J, Hildick-Smith D, Cotton J, et al. Contemporary clinical outcomes of patients treated with or without rotational coronary atherectomy - an analysis of the UK central cardiac audit database. Int J Cardiol 2014; 170: 381-7.

12. Sakakura K, Inohara T, Kohsaka S, et al. Incidence and determinants of complications in rotational atherectomy: insights from the National Clinical Data (J-PCI Registry). Circ Cardiovasc Interv 2016; 9: e004278.

13. Kawamoto H, Latib A, Ruparelia N, et al. In-hospital and midterm clinical outcomes of rotational atherectomy followed by stent implantation: the ROTATE multicentre registry. Eurointervention 2016; 12: 1448-56.

14. Édes IF, Ruzsa Z, Szabõ G, et al. Clinical predictors of mortality following rotational atherectomy and stent implantation in high-risk patients: a single center experience. Catheter Cardiovasc Interv 2015; 86: 634-41.

15. Eftychiou C, Barmby DS, Wilson SJ, et al. Cardiovascular outcomes following rotational atherectomy: a UK multicentre experience. Catheter Cardiovasc Interv 2016; 553: 546-53.

16. Généreux P, Campos CM, Yadav M, et al. Reasonable incomplete revascularisation after percutaneous coronary intervention: the SYNTAX Revascularisation Index. Eurointervention 2015; 11: 634-42.

17. Thygesen K, Alpert J, White H. Joint ESC/ACCF/AHA/WHF Task Force for the Redefinition of Myocardial Infarction. Universal definition of myocardial infarction. J Am Coll Cardiol 2007; 50: 2173-95.

18. Wu C, Camacho FT, King SB, et al. Risk stratification for longterm mortality after percutaneous coronary intervention. Circ Cardiovasc Interv 2014; 7: 80-7.

19. Pagnotta P, Briguori C, Mango R, et al. Rotational atherectomy in resistant chronic total occlusions. Catheter Cardiovasc Interv 2010; 76: 366-71.

20. Patel SM, Pokala NR, Menon RV, et al. Prevalence and treatment of "balloon-uncrossable" coronary chronic total occlusions. J Invasive Cardiol 2015; 27: 78-84.

21. Farooq V, Serruys PW, Bourantas CV, et al. Quantification of incomplete revascularization and its association with five-year mortality in the synergy between percutaneous coronary intervention with taxus and cardiac surgery (SYNTAX) trial validation of the residual SYNTAX score. Circulation 2013; 128: 141-51.

22. Genereux P, Campos CM, Faroog V, et al. Validation of the SYNTAX revascularization index to quantify reasonable level of incomplete revascularization after percutaneous coronary intervention. Am J Cardiol 2015; 116: 174-86.

23. Park KW, Kang J, Kang SH, et al. The impact of residual coronary lesions on clinical outcomes after percutaneous coronary intervention: residual SYNTAX score after percutaneous coronary intervention in patients from the Efficacy of Xience/Promus versus Cypher in rEducing Late Loss after stENTing (EXCELLENT) registry. Am Heart J 2014; 167: 384-92.e5. 